\title{
Verbraucherforschung besitzt einen Rahmen und Ziele
}

\author{
Christian Grugel
}

Published online: 29 July 2014

(C) Bundesamt für Verbraucherschutz und Lebensmittelsicherheit (BVL) 2014

In den letzten fünf Jahren hat die Verbraucherforschung einen Aufschwung erfahren. Gestützt auf die Vorstellung, dass in dem interdisziplinären Wissenschaftsfeld aus Rechts-, Wirtschaft- und Gesellschaftswissenschaften wesentliche Erkenntnisse der Verbraucherforschung anfallen, ist die Zusammenarbeit zwischen diesen Wissenschaftsgebieten ausgeweitet worden. Auf Bundesebene und in Nordrhein-Westfalen sind offene Netzwerke der in der Verbraucherforschung arbeitenden Wissenschaftler entstanden. Parallel zu dieser Entwicklung haben wissenschaftliche Gremien in der Verbraucherpolitik eine zunehmende Bedeutung erfahren. Unterstützt durch Mittel des Bundesministeriums für Ernährung, Landwirtschaft und Verbraucherschutz wurde an der Universität Bayreuth ein Lehrstuhl für Verbraucherrecht eingerichtet.

Die nach der Bundestagswahl 2013 gebildete Bundesregierung hat die Aufgaben des wirtschaftlichen und rechtlichen Verbraucherschutzes an das Bundesministerium der Justiz und für Verbraucherschutz verlagert. Im Koalitionsvertrag haben sich die Regierungsparteien darauf verständigt, einen „unabhängigen und interdisziplinär besetzten Sachverständigenrat für Verbraucherfragen “ zu bilden, der „zu wichtigen Verbraucherfragen und Teilmärkten Stellungnahmen und Empfehlungen formulieren“ soll. Dies stellt die Weichen für eine auf wissenschaftliche Erkenntnisse gestützte, evidenzbasierte

C. Grugel ( $\square)$

Bundesministerium der Justiz und für Verbraucherschutz, Leiter der Abteilung Verbraucherpolitik, Postanschrift:

Mohrenstraße 37, Berlin 10117, Germany

e-mail: grugel-ch@bmjv.bund.de
Verbraucherpolitik. Hierdurch wird auch der Rahmen für die Verbraucherforschung abgesteckt. Die Möglichkeiten der Verbraucherforschung verbessern sich, mit ihren Ergebnissen zur Gestaltung der Verbraucherpolitik beizutragen. Die Notwendigkeit, auf Erkenntnisse der Verbraucherforschung zurück zu greifen, ergibt sich schon aus den fundamentalen Änderungen, die mit der Entstehung der Informationsgesellschaft verbunden sind. Diese Änderungen lösen Megatrends aus und gestalten sie maßgeblich. Ihr Einfluss ist auch dann erheblich, wenn sie den einzelnen Megatrend nicht ausgelöst haben und nur mittelbar auf ihn einwirken. Diese Megatrends sind insbesondere die Globalisierung, die Entwicklung von Demographie und Migration, die Belastung der natürlichen Ressourcen (Umwelt, Natur, Klima), die begrenzte Verfügbarkeit von Rohstoffen und Energie sowie der Bedeutungszuwachs der Finanz- gegenüber der Realwirtschaft.

Auch die Verbraucherforschung muss auf die aktuellen Megatrends ausgerichtet werden. Stärker noch als andere Bereiche von Wirtschaft, Wissenschaft und Gesellschaft wird die Situation der Verbraucher durch die Entstehung der Informationsgesellschaft bestimmt. Wenn Verbraucher Rechnungen mit einer Check- oder Kreditkarte bezahlen, Geschäfte über das Internet abschließen oder Buchungen im Onlinebanking vornehmen, hinterlassen sie Spuren im Netz. Das geschieht auch, wenn sie mit anderen Personen über das Telefon, per E-Mail, in sozialen Netzwerken oder über andere Dienste im Internet Informationen austauschen. Die Spuren, die sie hinterlassen, bestehen nicht nur aus den Informationen selbst, sondern auch aus den Daten, zu welchem Zeitpunkt sie mit welchen 
Personen über welchen Dienst kommuniziert haben. Diese Metadaten erlauben es, das Netz von Kontakten und Beziehungen abzubilden, in dem sich das Leben der Verbraucher abspielt. Die regelmäßigen Signale, die Mobiltelefone und Smartphones an die Funkmasten der Telekommunikationsdienste senden und die Nutzung von GPS-Diensten ermöglichen es darüber hinaus, Bewegungsprofile der Verbraucher zu erstellen. Diese elektronischen Spuren im Netz werden von vielen Unternehmen der Internetwirtschaft personalisiert und zu Profilen zusammen geführt. Die Profile gestatten es, den Verbrauchern individuelle, an ihrem Lebensstil, ihren Konsumgewohnheiten und ihren wirtschaftlichen Möglichkeiten ausgerichtete Angebote zu machen. Die Profile liefern aber auch hervorragende Hinweise dafür, mit welcher Ansprache die individualisierten Angebote gemacht werden müssen, um von den Verbrauchern positiv wahrgenommen und akzeptiert zu werden. Der wirtschaftliche Erfolg personalisierter Werbung im Internet hat Werbung treibende Unternehmen dazu veranlasst, immer größere Teile ihre Aktivitäten aus klassischen Medien in das Netz zu verlagern. Die ständige Beobachtung der Aktivitäten von Verbrauchern im Netz und die Auswertung der daraus erstellten Profile stellt mittlerweile eine ernsthafte Herausforderung für die Verbrauchersouveränität dar. Dieser Herausforderung wird sich unsere Gesellschaft nur stellen können, wenn die Verbraucherforschung Erkenntnisse darüber liefert, welche Techniken manipulativ sind und welche Grenzen diesen Techniken gesetzt werden können.

Über viele Jahre ist die Verbraucherpolitik dem Paradigma gefolgt, dass die Transparenz der Angebote ausreicht, um den Verbrauchern selbstbestimmte, ihren Interessen dienende Entscheidungen zu ermöglichen. Dabei ist übersehen worden, dass die Verständlichkeit der Informationen und die Vergleichbarkeit der Waren und Dienstleistungen bei einem Übermaß an Informationen abnimmt. In einer immer komplexeren Welt kann es nicht gelingen, einfachere Produkte und Dienstleistungen zum Standard der Wirtschaft zu machen. Häufig ist die Komplexität eine inhärente Eigenschaft bestimmter Produkte und Dienstleistungen. Reduzieren lässt sich aber die Komplexität der Information. Umfangreiche Informationen über komplexe Angebote können auf wenige verständliche und vergleichbare Inhalte zurückgeführt werden. Werden Umfang und Komplexität der Informationen reduziert, führt dies auch $\mathrm{zu}$ einem Verlust an Informationen selbst. Eine zu große Vereinfachung ist irreführend. Die vollständigen Informationen über sehr komplexe Produkte und Dienstleistungen sind für viele Verbraucher oft wenig verständlich. Zumindest geht bei Informationen mit großem Umfang und hoher Komplexität die Vergleichbarkeit verloren. Dies gilt zum Beispiel für bestimmte Finanzprodukte, Dienstleistungsverträge mit hoher Regelungsdichte in den allgemeinen Geschäftsbedingungen oder umfassende Informationen über die Zusammensetzung und den Nährwert bestimmter Lebensmittel. Um den Verbrauchern verständliche und vergleichbare Informationen $\mathrm{zu}$ geben, ist eine Balance zwischen der notwendigen Reduktion und dem unverzichtbaren Inhalt von Informationen $\mathrm{zu}$ finden. Dabei muss sich die Verbraucherforschung an den intellektuellen und kognitiven Fähigkeiten der Menschen orientieren. Neben der Verhaltensökonomik muss sich die Verbraucherforschung hier auch auf die Neurowissenschaften stützen. Für die Forschung geht es darum, Grundsätze zu identifizieren, an denen sich alle Informationen für Verbraucher ausrichten sollten. Dabei darf es keinen Unterschied machen, welche Märkte betrachtet werden.

Spätestens seitdem das Buch „Nudge“ von Thaler und Sunstein in deutscher Sprache erschienen ist, wurde die Frage, wie man kluge Entscheidungen anstößt, auch außerhalb der Verhaltensökonomik diskutiert. Verbrauchersouveränität ist nur möglich, wenn die Verbraucher bewusste Entscheidungen treffen. Welche Entscheidungen sie treffen können, ist dabei ganz maßgeblich davon abhängig, welche Entscheidungsalternativen ihnen angeboten werden. Neben den konkreten Entscheidungsmöglichkeiten spielen insbesondere bei Vertragsabschlüssen im Internet oft auch Voreinstellungen eine wichtige Rolle. Die Anstöße für kluge Entscheidungen müssen, mehr noch als dies in der Vergangenheit geschehen ist, in den Mittelpunkt der Verbraucherforschung rücken. Dabei ist es wichtig, dass die Verbrauchersouveränität sowohl gegenüber der Wirtschaft wie auch gegenüber den Vorstellungen des Staates gewahrt bleibt. Die Verbraucherforschung sollte Wege aufzeigen, wie den von vielen Seiten unternommenen Versuchen begegnet werden kann, die Verbraucher in ihren selbstbestimmten Entscheidungen einzuschränken.

Nicht zuletzt muss sich die Verbraucherforschung auch mit der Frage beschäftigen, welche Rolle dem kollektiven Verhalten von Verbrauchern bei der Verhinderung oder Auflösung von Monopolen und Oligopole in der Wirtschaft zufällt. Dies wird umso dringlicher, je stärker einzelne Märkte, insbesondere im Bereich der Internet- und Telekommunikationswirtschaft, durch globale Monopole einzelner 
Anbieter bestimmt werden. Das Bundeskartellamt, das in der Vergangenheit stets als Behörde zur Bewahrung des Wettbewerbs in der Wirtschaft verstanden wurde, sieht sich heute auch als Verbraucherbehörde. Auch dies macht deutlich, dass die Aufgaben der Verbraucherforschung über den früheren Rahmen, in dem Verbraucherpolitik als
Reparaturbetrieb der sozialen Marktwirtschaft verstanden wurde, hinausgehen müssen. Verbraucherpolitik ist Wirtschaftspolitik aus der Perspektive der Verbraucher. Dieses Verständnis macht deutlich, welche Verantwortung und welche Möglichkeiten in der Verbraucherforschung liegen. 